Relations industrielles

Industrial Relations

\title{
Le manifeste communiste
}

\section{Egbert Munzer}

Volume 3, numéro 7, mars 1948

URI : https://id.erudit.org/iderudit/1023604ar

DOI : https://doi.org/10.7202/1023604ar

Aller au sommaire du numéro

Éditeur(s)

Département des relations industrielles de l’Université Laval

ISSN

0034-379X (imprimé)

1703-8138 (numérique)

Découvrir la revue

Citer cet article

Munzer, E. (1948). Le manifeste communiste. Relations industrielles / Industrial Relations, 3(7), 99-103. https://doi.org/10.7202/1023604ar

Tous droits réservés ( $@$ Département des relations industrielles de l’Université Laval, 1948
Ce document est protégé par la loi sur le droit d'auteur. L'utilisation des services d'Érudit (y compris la reproduction) est assujettie à sa politique d'utilisation que vous pouvez consulter en ligne.

https://apropos.erudit.org/fr/usagers/politique-dutilisation/ 
coutumières ou juridiques susceptibles de donner au salarié la certitude que son métier est bien à lui, qu'une stabilité, seule compatible avec sa dignité, lui est assurée, et qu'enfin nul ne pourra se prévaloir d'une situation s'il n'a pas la compétence requise.

D'un autre côté, il est bon de rappeler que le droit de propriété n'est jamais absolu. Son usage n'est moralement légitime et politiquement fructueux que s'il est mis au service de toute la communauté de travail.

B-La communauté de profit forme le centre des préoccupations que l'on résume généralement sous le nom de réformes de structure. Dans la mesure où le capital et le travail font partie d'une même communauté, il convient que les fruits de leur effort soient répartis entre les deux. Selon R. Kothen (3) voici comment les moralistes envisagent la répartition des parts:

a) Chaque partie a droit à sa «restitutio in integrum », c'est-à-dire que le capital doit pouvoir être reconstitué, et le travail doit être rémunéré en stricte justice;

b) Quant au surplus, il doit être partagé, en donnant un pourcent d'intérêt au capital investi prélevé sur les bénéfices, et une autre part des bénéfices doit être attribuée au travail.

Tel est le principe. Telles sont les exigences d'un véritable humanisme économique. Nous examinerons ultérieurement dans le détail les différentes solutions pratiques qui ont été proposées:

(3) R. Kothen, Problèmes sociaux actuels. participation aux bénéfices, participation aux fruits, salaire proportionnel, équipes autonomes, actionnariat ouvrier, etc.

C-La communauté d'autorité demeure le problème le plus délicat à traiter si l'on veut respecter à la fois l'équité et la délicatesse. D'une part il est certain que l'autorité doit être unique, sous peine de dégénérer en anarchie. D'autre part, l'autorité ne doit pas être arbitraire, elle ne doit pas ignorer systématiquement ou même par simple distraction les réactions et même les conseils de ceux sur lesquels elle s'exerce. Selon les dimensions de l'entreprise, selon qu'elle a le caractère d'une propriété familiale ou d'une société anonyme, selon enfin le degré de maturité des syndicalistes, des formules diverses peuvent être imaginées, depuis le simple délégué du personnel jusqu'au comité d'entreprise. En un tel domaine, la forme institutionnelle a moins d'importance que l'esprit qui l'anime. Le but à atteindre est de permettre, au sein de l'entreprise, des échanges fréquents entre personnel de direction et personnel d'exécution et le développement d'un climat de confiance réciproque.

Communauté de la propriété d'un même métier, communauté du profit résultant d'un même effort, communauté d'autorité par adhésion à une hiérarchie tempérée par l'esprit de collaboration, tels sont les réformes à opérer au sein de l'entreprise. Le premier et le troisième point résultent surtout d'un esprit, le second suppose le choix d'une technique. C'est à lui que nous consacrerons notre prochaine étude.

\section{AUTOUR D'UN CENTENAIRE}

\section{LE MANIFESTE COMMUNISTE}

\section{Egbert MUNZER}

Il y a exactement un siècle aujourd'hui, Marx et Engels publièrent à Londres, en langue allemande, le «Manifeste du Parti communiste». C'était en février 1848. Peu de jours après la publication du «Manifeste», la Révolution éclatait à Paris.

A l'été de 1847, un groupe d'artisans, Allemands pour la plupart, tinrent à Londres le premier congrès de la «Ligue communiste». Engels était présent. C'est lui qui proposa l'élaboration d'un programme qui servirait de base à un congrès futur de la «Ligue». Il rédigea un «catéchisme» composé de vingt-cinq questions qu'il soumit, conjointement avec Marx, au second congrès de la «Ligue», en novembre 1847. Ce sont les délégués de ce second congrès qui chargèrent Marx et Engels de la rédaction d'un «Manifeste » basé sur les vingt-cinq points d'Engels. Marx composa le Manifeste durant les mois d'hiver de 1847-48. Ce texte allait devenir l'un des plus célèbres documents du siècle.
Le Manifeste se divise en quatre parties. La première contient un précis d'histoire, telle que Marx, la concevait. Selon Marx, l'histoire, c'est l'histoire de la lutte des classes, du combat incessant entre deux couches de la société humaine, une classe opprimante et une classe opprimée. Durant un millénaire, la classe féodale domina la société européenne et l'Histoire. Au XVIIe et au XVIIIe siècles, d'abord en Angleterre, puis ensuite en France, la classe féodale fut détrônée par la bourgeoisie.

La bourgeoisie mit sur pied «des forces de production puissantes et colossales», et changea la face du monde. Mais ces forces « ont maintenant pris de telles proportions, qu'elles ne sont plus compatibles avec les relations de la propriété bourgeoise et la suprématie bourgeoise comme classe dominante». La nouvelle classe révolutionnaire, c'est celle des ouvriers de l'industrie, c'est le prolétariat. Le prolétariat se servira de la révolution pour enlever à la bourgeoisie sa propriété et sa 
domination politique, pour mettre fin à l'exploitation de l'homme par l'homme au moyen de la socialisation de tous les moyens de production - restaurant ainsi à jamais l'harmonie sociale.

La seconde partie du Manifeste fournit quelques détails sur la ligne de conduite du nouveau «Parti communiste». Il s'agit ici de la partie la plus progressive et la plus active de la classe ouvrière de tous les pays. Son objectif premier, c'est de fomenter des révolutions qui seules mettront fin à la domination bourgeoise, feront du prolétariat la nouvelle couche sociale dominante et, finalement, aboliront l'Etat.

Danș la troisième partie, Marx fait la critique d'autres formes du socialisme, qu'il dénonce comme ou peu sincères ou utopiques. La dernière partie est une façon d'épilogue: elle traite des relations des communistes avec d'autres révolutionnaires, et constitue par essence une continuation de la polémique entreprise dans la troisième partie de l'oeuvre.

Pour la forme extérieure, le contenu et la méthode logique, le Manifeste n'est pas sans précédent. Dans sa forme, il est beaucoup plus une sorte d'incantation, d'admonition religieuse qu'une analyse scientifique. Ses cadences psalmodiques révèlent les réminiscences psychologiques de l'Ancien Testament et du calvinisme, avec leur quasi-pharisaïsme et leur soif bruyante de justice; elles rendent compte de forces religieuses subconscientes dans Marx et dans Engels, forces qui servent pour des fins politiques et qui se pervertissent dans des visées antireligieuses ou ireligieuses.

Le contenu du Manifeste était dans l'air depuis la Révolution française, qui avait entraîné une certaine liberté politique, mais qui n'avait pu établir l'égalité sociale et économique. Babeuf, dans sa «Conspiration des Egaux», Fourier et Owen, avec leurs idées sur un communisme plutôt idyllique, Proudhon, dans son identification des lois économiques et des lois historiques, enfin Blanqui et d'autres, avec leur interprétation de l'histoire sous l'angle des classes, - tous ces individus et beaucoup d'autres pourraient être mis à contribution pour réduire les prétentions de Marx à l'originalité et l'installer sur un piédestal plus modeste que celui que ses successeurs voudraient bien lui édifier.

Enfin, la méthode historique appliquée dans le Manifeste est clairement celle de Hegel: l'histoire comme champ de bataille de la dialectique, de la thèse, de l'antithèse et de la synthèse. Avec cette différence près, cependant, que les classes, groupes d'être vivants, prennent la place des idées semi-divines du «praeceptor mundi » qu'a été le sibyllin professeur allemand.

Mais, même si d'amples déductions ont été faites à partir des prétentions de Marx à la création véritable, la profonde influence du Manifeste ne peut être déniée que par ceux qui aiment à se fermer les yeux devant la réalité sociale et historique.

Au cours du siècle qui a suivi sa promulgation, le Manifeste a été mis à l'épreuve en face de l'arrière-plan de l'évolution historique, et aujourd'hui, sa valeur intrinsèque peut s'estimer avec un certaine précision. Mais avant de tenter une appréciation de ce genre, il nous faut poser un bref jugement sur l'idée fondamentale qui soutient le Manifeste.

Cette idée que l'histoire est déterminée exclusivement - ou même principalement - par l'antagonisme et la lutte ouverte entre les classes sociales est évidemment inacceptable pour l'idéologie chrétienne, et de même pour la pensée religieuse en général. Cette idée équivaut à une laicisation totale de l'histoire; elle ne laisse aucune place aux forces qui transcendent le processus historique visible; en fait, elle n'est rien d'autre qu'une forme particulière d'athéisme. Elle vient en contradiction avec la morale religieuse, qui voit dans les luttes et les inimitiés de classe des symptômes d'un malaise moral collectif plutôt que les forces normales et régulières qui font l'histoire.

Il peut paraître surprenant qu'une telle doctrine areligieuse de l'histoire puisse influer sur une bonne partie de l'humanité, et cela, d'une manière plus profonde que toute autre philosophie nonchrétienne. Mais c'est précisément le caractère pseudo-religieux du Manifeste - et des travaux subséquents de Marx et d'Engels - qui a engendré des effets aussi puissants. Le Manifeste fait appel aux sentiments religieux des hommes pour des intentions et des fins qui cessent d'être authentiquement religieuses. Particulièrement en Marx se localise un curieux mélange entre le savant économico-social et le prophète ou le chef charismatique, quoique d'un genre fortement laïcisé.

Nous pourrions même aller jusqu'à appeler Marx le « Jérémie du XIXe siècle », avec ses violentes dénonciations de l'exploitation capitaliste, ses admonestations sermonneuses à un monde sombre et galeux, et enfin la prédiction qu'il a 
faite d'un Age d'Or où tous les antagonismes s'apaiseraient. Chez Marx comme chez Engels, des forces religieuses subconscientes - particulièrement celles de l'Ancien Testament - étaient en fermentation; et ce sont ces forces qui façonnèrent en dernier ressort leurs idées-mères : le prolétariat représente le Peuple Elu; les capitalistes, ce sont les Gentils; à l'instar des Egyptiens, des Babyloniens et des Assyriens, ils oppriment la Race Choisie; la Révolution à venir sera le Jour du Jugement. C'est là que le Peuple Elu triomphera des iniquités et des vicissitudes de ce monde. Le Paradis sur terre, le Royaume de Dieu bien établi sur le globe terrestre, la laicisation des fins et des sentiments religieux - voilà, en dernière analyse, la note caractéristique des prédictions de Marx et la justification de l'activité révolutionnaire.

Et c'est cette contrefaçon de religion, et non pas les analyses scientifiques de Marx, qui a captivé les générations d'humains depuis 1848 . Voilà un fait étrange, dont nous pouvons tirer d'ici au moins une conclusion principale : seule une religion authentique, et non pas la science, triomphera en dernière analyse des abberrations religieuses du matérialisme historique.

Il nous faut donc répudier la philosophie de l'histoire qui sert de base au Manifeste - et à son fils spirituel, le communisme moderne. Il nous faut cependant reconnaître les vérités partielles que renferme le Manifeste, à l'instar des hérésies religieuses. On ne peut dénier l'existence de tensions sociales; autrement, les révolution sociales seraient inimaginables. A des époques données, des créations religieuses et culturelles détermineront l'histoire, et les querelles religieuses donneront naissance à des cataclysmes sociaux. A d'autres époques, les forces économiques et sociales auront une signification plus grande que les valeurs religieuses et spirituelles. Bien plus, il peut être vrai d'affirmer que, dans l'histoire moderne, les forces économiques tendent sans cesse à surpasser les autres forces dans leur signification historique. Mais ce fait ne signifie pas qu'il en a toujours été ainsi, ou qu'à l'avenir il en sera toujours ainsi.

Le fait que le Manifeste est lui-même une création de l'histoire soumise à la relativité historique devient évident, si maintenant nous mettons le Manifeste à l'épreuve en le considérant en regard d'un siècle d'expériences sociales. Dans l'ensemble, l'ouvrage contient deux simplifications trop poussées, essentielles à la philosophie marxiste, inais qui ne répondent pas aux exigences de l'épreuve historique; ce sont: la prétention qu'il y a toujours eu deux classes sans cesse en guerre ouverte, et l'insistence de Marx sur le processus d'évolution à partir du régime féodal, en passant par le stage nécessaire du capitalisme bourgeois, pour aboutir enfin au paradis prolétarien.

La première simplification peut avoir son explication dans l'intérêt spécial que portait Marx aux conditions existantes en France et surtout en Angleterre. Dans ces deux pays - mais seulement là - le système féodal avait croulé sous la révolution. De plus, l'Angleterre ne comptait pour ainsi dire plus de classe paysanne, de sorte que les ouvriers et les capitalistes bourgeois paraissaient former les deux couches essentielles de la société. Par contre, il existait en France une classe paysanne aux effectifs fort considérables; et ce fait se vérifiait - et se vérifie encore - dans tous les pays du continent, pour ne rien dire des nations non-européennes.

Marx était prêt à reconnaître l'existence d'une petite-bourgeoisie qui oscillerait entre les capitalistes et les ouvriers. Cependant, cette concession est à peine suffisante. L'histoire du siècle qui s'est déroulé entre 1848 et 1948 a démontré que les hommes, à moins d'être contraints durant quelque temps de se laisser enfermer dans les deux enclos que la sollicitude de Marx a prévus pour leur bienêtre et leur conformité avec les desseins de Hegel. Les hommes aspirent à plus de variété. Les recherches modernes, et en particulier celles de l'éminent savant italien Mosca, ont bien montré qu'il existe une classe dominante - qui d'ailleurs n'est même pas homogène au point de vue économique - et une masse asservie, qui est tout normalement partagée en un certain nombre de couches.

La question de la classe paysanne a constitué la première grande lacune du Manifeste. Depuis, les tendances de la société à se stratifier de plus en plus se sont accrues : par exemple, le fonctionnarisme moderne dans les pays européens superbureaucratisés et en Russie est devenu une couche qui tend à remplacer la classe féodale de jadis.

C'est un fait bien connu que Lénine, en 1903, élabora un nouveau plan d'après lequel le prolétariat, à peine existant en Russie, où 80 pour cent de la population d'alors étaient des paysans, devait s'emparer du pouvoir avec l'aide des paysans, puis établir une «dictature démocratique» d'ouvriers et de paysans. Mais l'évolution actuelle de la Révolution russe a démontré à l'évidence que cette amalgamation ne pouvait mener qu'à la prolétarisation délibérée des paysans, qui sont aujourd'hui devenus des ouvriers dans ces « usines 
à grains » que l'on nomme kolkhozes et sovkhozes. Du plan d'histoire sociale, au sens de Marx, il ne reste plus rien.

L'autre grande faiblesse du point de vue quavait Marx du développement historico-social est en étroite relation avec son postulat du cycle des deux classes. Şelon les données de Marx - et du sens commun - le prolétariat ne peut s'emparer du pouvoir que s'il existe, et il ne peut exister qu'à la suite du développement du capitalisme et de la formation d'une bourgeoisie qui soit la classe politique dominante. Mais cette classe capitaliste-bourgeoise peut bien - comme c'est le cas dans le monde slave - ne pas exister du tout; ou encore elle peut être incapable ou pas désireuse du tout de faire sa propre révolution comme ce fut le cas en Allemagne jusqu'à 1918, et même dans la suite. Dans ces deux cas, le système marxiste ne s'applique pas. Marx n'a jamais ajusté sa doctrine à ces faits - «tant pis pour les faits », se serait exclamé son père philosophique Hegel!

En 1859, dans sa Préface à la «Critique de l'Economie politique », Marx répétait: «Il n'est pas de forme sociale qui disparaisse avant que toutes les forces de production qu'elle contenait en puissance aient été développées ». Ce n'est que vers la fin de sa vie que Marx admit - en tant que chef de parti et malgré sa «science» - qu'une nation paysanne comme la Russie pouvait à la rigueur accomplir la révolution sociale sans passer par la phase capitaliste-bourgeoise. C'est ainsi qu'il écrivit dans la Préface à la traduction russe du Manifeste publiée en 1882: «Si la révolution russe donne le signal d'une révolution ouvrière en Occident, et que toutes deux se complètent, la propriété commune (obstchina) actuelle de la Russie pourra servir de point de départ à une évolution communiste ».

La Révolution bolchéviste ne fut pas le «signal d'une révolution générale des ouvriers, mais elle introduisit le communisme en Russie. Et elle le fit en flagrante contradiction avec l'analyse révolutionnaire du Manifeste. Une fois de plus, c'est Lénine, de concert avec ses collaborateurs bolchévistes, qui a développé «créativement»le marxisme au point qu'il ne restait plus rien du Manifeste, si ce n'est un cadre, absolument vide en ce qui concerne la Russie, et pauvrement rempli en ce qui touche les pays occidentaux.

On sait que la division entre les bolchévistes et les menchévistes s'est opérée, lors du fameux congrès de Londres en 1903, au sujet de l'interprétation du Manifeste et de l'élaboration subséquente de la doctrine de Marx sur l'histoire. Les menchévistes, menés par le lumen théorique Plekhanoff, mirent l'accent sur l'analyse marxiste: ils considérèrent la création d'une puissante industrie capitaliste et d'un régime bourgeois en Russie comme la condition nécessaire et pré-requise de la future révolution prolétarienne. Les bolchévistes différèrent d'avis, et Lénine élabora le «Nouveau Testament» du communisme dans le sens que l'on a expliqué plus haut. Comme résultat plutôt surprenant, Marx, le premier Saint Patron du communisme russe, doit ètre considéré comme un menchéviste, et non comme un «marxiste orthodoxe». Et ceci s'applique à Engels, dans une plus grande mesure encore. C'est plus que probable que leurs disciples russes les auraient «liquidés», sous prétexte de «trahison contre le Marxisme », si une mort bienheureuse dans l'Angleterre de Victoria ne les avait préservés depuis longtemps de la gloire pas tellement convoitée du martyre.

Ces deux importantes contradictions entre ia philosophie exprimée dans le Manifeste et la réalité sociale ne peut qu'affecter gravement la solidité des fondations principales sur lesquelles Marx a édifié sa tour. Le Marx du Manifeste est sûr, absolument sûr que la prise du pouvoir politique par le prolétariat sera un acte révolutionnaire. Plus tard, cependant, Marx lui-même cessa de croire à cet article de foi. Il admit que, dans certains pays à civilisation avancée, la victoire du prolétariat pouvait s'obtenir sans violence révolutionnaire. Mais dans ces circonstances, que signifie «victoire du prolétariat»? Ce ne peut être que ceci : les membres du prolétariat monteront graduellement, et s'infiltreront dans la couche dominante, pour obtenir dans le gouvernement une part égale à l'importance sociale de la classe ouvrière industrielle. Une fois de plus, ce n'est ici que du sens commun : et on n'aurait pas eu besoin d'un Karl Marx pour le démontrer. Tous ceux qui ont analysé la société humaine, depuis Aristote jusqu'à Mosca, Sorel et Pareto, se rejoignent dans cette vérité.

En fait, dans toute l'Europe occidentale, l'absorption mutuelle de la classe dominante et des masses ouvrières semble présentement être à s'effectuer «sans violence révolutionnaire». Et c'est un peu déconcernant pour les Marxistes de constater que les pays en question sont précisé- 
ment les pays industrialisés, tels que l'Angleterre, où l'on pourrait trouver quelque ressemblance avec le système des deux classes de Marx et sur laquelle Marx s'est surtout fondé pour élaborer sa doctrine de la révolution.

D'un autre côté, les véritables révolutions sociales ont lieu dans les pays agraires de l'Europe orientale et de l'Asie. Pour ces pays, le Manifeste ne peut constituer une philosophie de l'histoire possédant même un degré modéré de précision; il peut être tout au plus une lumière quasi-religieuse et plutôt diffuse pouvant servir à l'établissement dun minimum de justice sociale.

Dans ces pays, les idées de Marx sont maintenant appliquées pour justifier une certaine sorte de capitalisme d'Etat, et pour les développer dans le sens du plan industriel établi par l'Europe occidentale et les Etats-Unis. Et pour couronner cette montagne d'inconsistence : en Russie, et dans tout le monde slave, le communisine se transforme de plus en plus en un nationalisme panslaviste violent qui doit faire frémir les restes de Marx dans son tombeau.

\section{THE COMMUNIST MANIFESTO}

\section{Egbert MUNZER}

Exactly a century ago today, Marx and Engels published in London, in the German language, the «Manifesto of the Communist Party». It was February 1848, and a few days after the publication of the «Manifesto», the revolution broke out in Paris.

In Summer 1847 a group of craftsmen, mostly Germans, held the first congress of the « Communist League » in London. Engels attended the congress, and it was he who proposed to draft a programme for a future congress of the «League». He composed a so-called catechism of twenty-five questions which he and Marx submitted to the second congress of the «League » in November 1847. This second congress charged Marx and Engels to draft a «Manifesto » on the basis of Engels' 25 points. Marx composed, during the winter-months $1847 / 48$, the Manifesto. It was to become one of the most-spoken-of documents of the century.

The Manifesto is divided into four parts. The first contains a «précis » of history as Marx saw it. History, to him, is the history of class-war, of the incessant contest between two strata of human society, a ruling and a subjected class. For a thousand years, the feudal class dominated European society and history. In the 17th and 18th century, first in England, then in France, the feudal class was overcome by the bourgeoisie. The bourgeoisie unleashed « powerful and colossal productive forces » and changed the face of the world. But these forces «have now grown to a point where they are no longer compatible with bourgeois property relations and bourgeois supremacy » as a ruling class. The new revolutionary class is the industrial workers, the proletariat. It will take over, by way of revolution, the property of the bourgeoisie and its political domination, and end the exploitation of man by man through the collectivization of all means of production; and thus restore social harmony forever. The second part of the Manifesto gives a few details of the policy of the new «Communist Party 》. It is the most progressive and activist section of the working class of all countries. Its main aim is the promotion of revolutions which alone will end bourgeois domination, establish the proletariat as the new ruling stratum, and finally abolish the State altogether. The third part contains a criticism of other forms of socialism, which are lumped together as being either insincere or utopian. The last part is some kind of epilogue on the relations of the communists to other revolutionaries and essentially a continuation of the polemic of the third section.

As to exterior form, material content and lo. gical method, the Manifesto is not without precedent. Its form is that of religious incantation and admonition rather than of scientific analysis. Its psalmodic cadences reveal the psychological residue of Old Testament and Calvinist selfrighteousness and clamour for justice. That is, subconscious religious forces in Marx and Engels are used for political purposes and perverted towards non-religious or a-religious ends. The contents of the Manifesto were « in the air» since the French Revolution which had brought some kind of political freedom but had failed to create social and economic equality. Babeuf, with his « Conspiration des Egaux», Fourier and Owen with their ideas of a rather idyllic communism, Proud- 